\title{
MAP OF THE MOUNT GONGGA GLACIER: A COMBINATION OF TERRESTRIAL AND AERIAL PHOTOGRAMMETRY
}

by

Chen Jianming

(Lanzhou Institute of Glaciology and Geocryology, Academia Sinica, Lanzhou, China)

\section{ABSTRACT}

For use in glaciological research, between 1982 and 1984 , we succeeded in surveying and mapping the Mount Gongga Glacier, on a scale of $1: 25000$, by means of a combination of terrestrial and aerial photogrammetry. This paper describes the method in detail.

In the survey area, we set up an independent, triangulation network, with microwave distance measurement, and two, independent, straight-line traverses, for basic control. Control points were observed by intersection. The terrestrial, photogrammetric baselines were projected and corrected into distances on the plane of the map.

Terrestrial photography accounted for the majority of the photographs of the survey area. Surveying and mapping of planimetric and topographic features were completed on a stereo-autograph, using plates mainly from terrestrial photogrammetry. Where these data were insufficient, they were supplemented by aerial photography, plotted on a photographic plotting instrument. Orientation points of the aerial photographs were established by terrestrial, photogrammetric analysis and located on the map by an optical, mechanical method.

The practical result showed that a combination of terrestrial and aerial photogrammetry, in mapping a high, mountain, glacier area, on a large scale, is more feasible and flexible than other methods and more economical as well.

\section{INTRODUCTION}

Mount Gongga, one of the most famous, high $(7514 \mathrm{~m})$, peaks in the world, lies in the middle section of the Daxue Shan, in the Hengduan Mountains, along the eastern end of the Qinghai-Xizang Plateau (Fig.1.).

From 1982 to 1984 , we successfully surveyed and mapped the Gongga Glacier on a scale of 1:25000, with a contour interval of $20 \mathrm{~m}$, by a combination of terrestrial and aerial photogrammetry.

The reasons for mapping the Mount Gongga Glacier were, first, to depict the physiographic character of this relatively maritime glacier in the eastern part of the Qinghai-Xizang Plateau for use in scientific research, as well as mountaineering and tourism, and, secondly, to test the feasibility of a combination of terrestrial and aerial photogrammetry for surveying and mapping a glacier area, as a research project.

The main instruments used in our survey and mapping were two Zeiss $1318 / 19$ photo-theodolites, two Zeiss 010 theodolites, microwave distance-measuring instruments, type $\mathrm{CW}-1$, a stereo comparator, a stereo-autograph, Topocart-B, and ultra-violet photo-plates. The aerial photographs were taken in the 1960 s

This paper describes in detail the combination of terrestrial and aerial photogrammetry used in surveying and mapping the Mount Gongga Glacier.

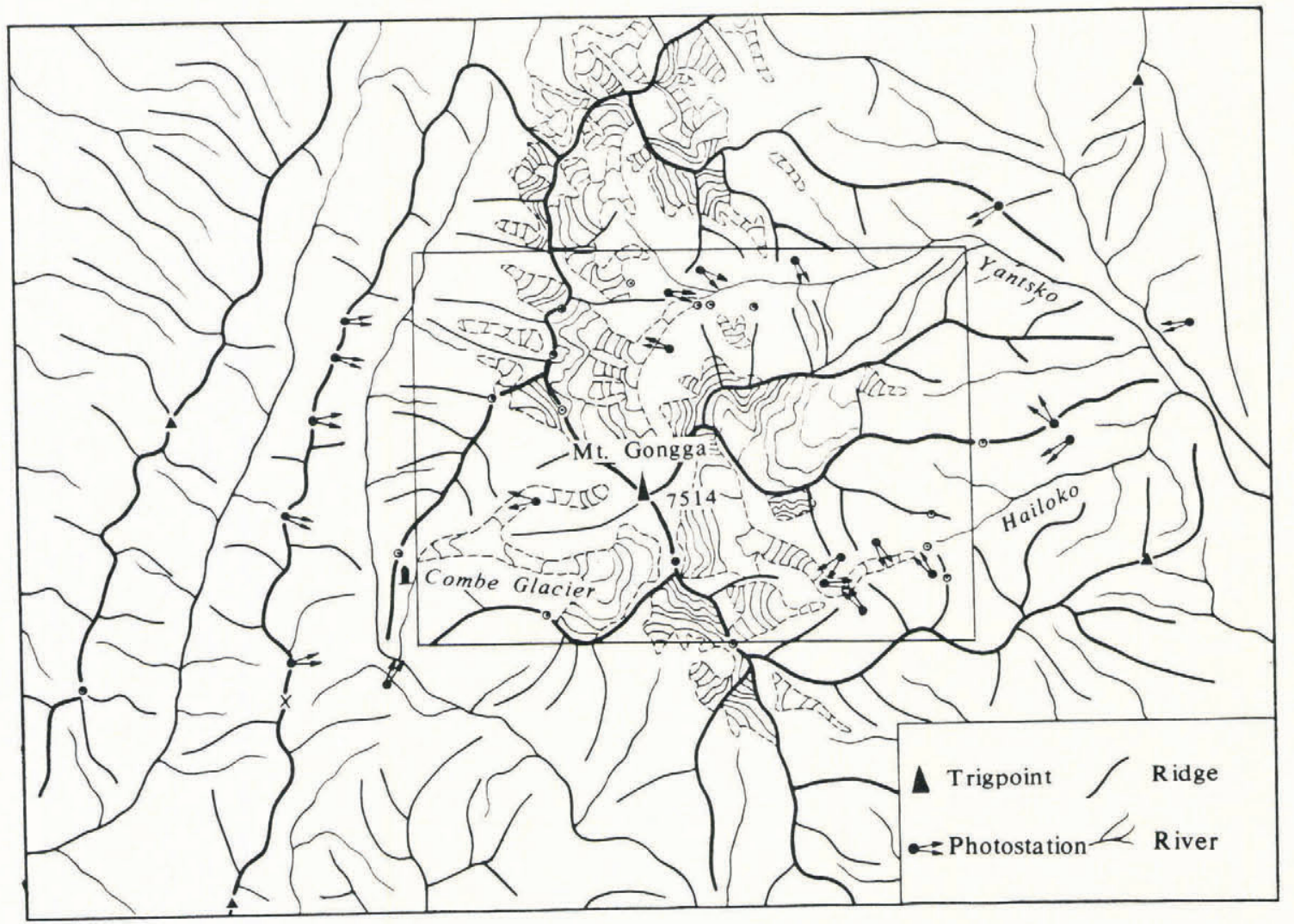

Fig.1. Map showing the photostations and trigpoints in the Gongga Area. 


\section{GENERAL SITUATION OF THE SURVEY AREA}

In the Mount Gongga region, the mountain blocks are high and precipitous, with deeply-cut, V-shaped gullies and valleys and relative height differences on the mountain slopes between 2000 and $3000 \mathrm{~m}$. Precipitation is concentrated in the period from June to September and is typically heavy and prolonged. The vertical zoning of vegetation is very marked. Communications are very difficult. Materials and equipment have to be carried on the backs of porters. Some glacier termini penetrate below the tree line a distance of $4 \mathrm{~km}$. Firn basins (at 5100 to $5500 \mathrm{~m}$ ) are very hard to climb.

It is well known that terrestrial photogrammetry offers many advantages for research work in glaciology. Its disadvantages are, first, that the positions of photo-stations must be determined in the field and a large number of checking points is necessary, thus involving much field work, and, secondly, the field of view may be partly obscured by mountains or ridges, causing gaps in the photographic coverage. In view of these factors, mapping the Mount Gongga Glacier is very difficult, if only terrestrial photogrammetry is used.

However, when aerial photographs are used, the very steep mountains and deep valleys may cause problems. Certain parts of the mountain slopes may not be photographed clearly enough for photogrammetric construction, while large distortions and natural shadows in valleys also add to the difficulties. Therefore, a combination of terrestrial and aerial photogrammetry proved very useful for the large-scale mapping of the Gongga Glacier.

The feasibility of mapping the Gongga Glacier by a combination of terrestrial and aerial photogrammetry was recognised after an analysis of the aerial photographic information. Within the required limits of accuracy and with a maximum magnification of the aerial photographs of 3.4 times, typical stereo instruments can be used to plot planimetric and topographic features and draw up contour lines.

\section{$\begin{array}{llllll}\text { SURVEY } & \text { WORK } & \text { FOR } & \text { MAPPING } & \text { THE } & \text { GONGGA }\end{array}$}

The principles of terrestrial and aerial photogrammetry are widely known. Only a few key stages in the mapping of the Gongga Glacier are discussed.

\section{Combined terrestrial/aerial photogrammetry programme}

The first essential stage is to work out an operational programme of office plotting and field work to suit the mapping purpose, accuracy requirement, size of surveyed area, type of instruments, etc.

In the Gongga area, the surveying and mapping of planimetric and topographic features were to be completed on a stereo-autograph, with data from terrestrial photography. Photographic gaps were filled with data from aerial photography, but the orientation points of the aerial photographs were determined by an analytic, terrestrial, photogrammetric method.

The field programme consisted of some basic mapping of survey control points and the terrestrial photography. Its basic requirements were that the established control points must be advantageous for the combined programme.

\section{Control survey}

The mathematical accuracy of the map depends on control points. There is no control point in the survey area, but two, trigonometric points, corresponding, respectively, to the first-order and second-order triangulation network, are located on the eastern and western slopes, about $20 \mathrm{~km}$ from the Gongga main ridge, outside the area of the map. From these two points, a control network was extended to the survey area, with fourth-order control surveying specification. The survey area was divided into two parts, east and west, by the main mountain chain, with an average height of over $6000 \mathrm{~m}$. Thus, the control network in the east is not directly connected with the western system, but both are in the same coordinate system. Independent, straight, traverse lines were laid out separately at Hailoka and Yantska, on the eastern slope of Gongga and an independent triangulation network was set up on the western slope. A combination of triangulation and trilateration methods was used. The height of the control points was determined by trigonometric levelling, both forward and reverse. All checking points (24 in number) were chosen from predominant summits, including the highest peak, Mount Gongga itself. Forward intersection was used in this
work.

The positional error of the weakest control point was \pm $0.3 \mathrm{~m}$, while the mean square error of weight unit in the trigonometric levelling was $\pm 0.0247 \mathrm{~m}$. The estimation of error in the positions of checking points was $\pm 0.7 \mathrm{~m}$ and the error in height was $\pm 0.3 \mathrm{~m}$. The position error of the summit of Mount Gongga is $\pm 2 \mathrm{~m}$ and the error in the determination of its height is $\pm 1 \mathrm{~m}$.

\section{Terrestrial photography}

The topography of the western slope of Gongga is comparatively open. Photographic baselines, distributed along the ridge of the ZiMei Shan, were ideal, with maximum photogrammetric distance, extensive range of view and few photographic gaps. The landscape on the eastern slope of the mountain is quite complicated, however, and is, for photogrammetric purposes, very difficult, with narrow gullies, deep and precipitous valleys and thickly forested areas, which are very difficult to climb. As a result, photogrammetric bases had to be located in the lower parts of the slope. With great difficulty, 23 photogrammetric bases were selected, but there were large photogrammetric gaps in the firn basin (Fig.1.). Photographic stations were surveyed directly in the field. More than one third of the photogrammetric bases were connected directly with control points and about one third were located by resection, with a mean positional error of $\pm 0.53 \mathrm{~m}$ and a height error of \pm $0.27 \mathrm{~m}$.

Here, the measured length of the photogrammetric bases is a horizontal distance (Wang Wenying, Chen Jianming 1980) and, in practical use, the length of a photogrammetric base whould be its length on the map plane, i.e. in the Gaussian plane, except in the case of very small areas of large-scale mapping.

\section{Photogrammetric control extension}

In both terrestrial and aerial photographic mapping procedures, control points in the photographs are needed, in order to achieve absolute orientation of the stereo models. An essential feature of a combined programme is to extend control points for the aerial photographs by using data from terrestrial photography. Thus, the accuracy of the combined method depends to a great extent on the accuracy of photogrammetric control points. The main reason why the combined method has not been used much before is that data for extending the computation of control points were only treated with standard, general, photogrammetric formulae and elements of interior and exterior orientation, which greatly affect the accuracy, were not corrected, resulting in poor accuracy of location of the control points. Therefore, generally, the relative error in distance from camera of photogrammetric points is less than 1: 1000 , which cannot meet survey requirements with a high degree of accuracy. In order to improve extensional accuracy, it is necessary to correct the error in elements of interior and exterior orientation by using an approximation of a closed, analytical method.

Analysis of the error theory for terrestrial photogrammetry shows that the error, dy, of the photogrammetric, longitudinal coordinate, Y, is always larger than the error of the latitudinal coordinate, $X$, and the height, $\mathrm{H}$, and that the dy error, affected by the dp error of the horizontal parallax, is the largest. Moreover, the dp error, affected by a convergent angle, $r$, is especially large; if $X_{L}=60, p=10, z=40, f=200 \mathrm{~mm}$, then $(d p)_{r}$ : $(d p)_{\alpha}:(d p)_{w}:(d p)_{k}=39: 1: 3: 10$. Thus, we see that the accuracy of a photogrammetric point can obviously be improved as soon as the error of convergence has been corrected.

The corrections affected by convergence were computed from the geodetic coordinate of a ground control point. According to error theory, the following formula is obtained: 


$$
d p=\frac{y}{p}\left(f+\frac{X_{R}^{2}}{f}\right) \frac{r}{\rho^{\prime \prime}}
$$

$r$, from the longitudinal coordinate of a ground point, is:

$$
\frac{r}{\rho^{\prime \prime}}=-\frac{f}{f^{2}+X_{o}^{2}} \cdot \frac{p_{o}}{y_{0}} \Delta y_{0}
$$

Substituting in the dp equation, this gives the correction of the longitudinal coordinate, as follows:

$$
\Delta y=\frac{f^{2}+X_{R}^{2}}{f^{2}+X_{R o}^{2}} \cdot \frac{y^{2}}{y_{0}^{2}} \Delta y o
$$

Similarly:

$$
\Delta X=\frac{X_{L}}{f} \Delta y \text { and } \Delta H=\frac{{ }^{z_{L}}}{f} \Delta y
$$

where $\mathrm{y}$ is the uncorrected, photogrammetric, longitudinal coordinate, $y_{0}$ is the photogrammetric, longitudinal coordinate of a ground control point, which may be found by a coordinate transformation, let $\Delta y_{0}=y_{0}-y_{o}^{1}$, and $x_{L}$, $\mathrm{X}_{\mathrm{p}}$ and $\mathrm{z}_{\mathrm{L}}$ are photographic coordinates on the left and right photographs.

A correction of the error of convergence by only one ground control point cannot be done satisfactorily, as it can only correct the error in the main item. But errors arise from other causes than the convergent angle itself and may actually introduce convergence error. Therefore, it is desirable to use more than two ground control points, in stereo pairs, in order to facilitate analysis of the main cause of error and to improve the accuracy and reliability of the extension points.

The author has used another method for computing the error of convergence (Lanzhou Institute of Glaciology and Geocryology 1980), in which the difference value between angles of both direction in the photo-optic axis and the ground control point is calculated, the homologous, horizontal angle from the terrestrial photograph is measured and then the error of convergence is computed from the angle of horizontal swing from left and right stations. This method can correct, not only the error of convergence, but also the error of angle of horizontal swing.

Using the methods described above, the accuracy of extension was sufficient for mapping the Gongga Glacier with the combined photogrammetric method. If one wanted large-scale mapping, covering several thousand square kilometres, with a combination of terrestrial and aerial photogrammetry, it would be necessary to use a strict, analytical, terrestrial photogrammetric method. The coordinate accuracy of photogrammetric points can reach 1:40 000 against photogrammetric distance by solution with 4 ground control points (Wang Zhizhuo 1979), or, by correcting from elements of the exterior, measured in the field, the relative error of photogrammetric distance can be as little as 1:100000 (Wang Zhizhuo 1979). However, many problems remain to be solved, such as the relative improvement in accuracy of photo-theodolites and other instruments in the office, raising the power of resolution of photographic materials, etc.

\section{CONCLUSIONS}

The results of surveying and mapping the Mount Gongga Glacier showed that the use of a combination of terrestrial and aerial photogrammetry, in mapping high, mountain, glacier areas, on a large scale, is not only feasible, but also advantageous. Field work for terrestrial photography and terrestrial photogrammetric control extension are the two, central, key links in the chain of execution of the combined mapping programme. They can raise the mapping accuracy and also lower the cost of the survey.

\section{REFERENCES}

Lanzhou Institute of Glaciology and Geocryology 1980 The expedition and study on the Batura glacier in Karakoram mountains. Lanzhou

Wang Wen-Ying, Ch'en Chien-Ming 1980 Terrestrial stereophotogrammetric surveying and mapping in the region of Mount Qomolangma and the Batura glacier in Karakoram. By Wang Wenying and Chen Jianming. Journal of Glaciology and Cryopedology 2(4): 22-28

Editor's note: Titles quoted above are English versions of the original Chinese. 